The patient, a 6-year-old boy, had absence attacks precipitated by gazing at window screens or patterns of vertical, but not horizontal, parallel black and white lines. He also was sensitive to intermittent photic stimulation. While photosensitive and pattern-sensitive epilepsy share common characteristics, subtle differences are noted. Pattern-sensitive patients without photosensitivity have a higher incidence of focal symptomatic epilepsies, neurologic abnormalities, and focal EEGs (Brinciotti M et al. Epilepsia 1994;35:842-849; Ped Neur Briefs Nov 1994). The EEG in pattern-sensitive children shows focal epileptiform discharges in occipital regions, whereas photosensitive patients have generalized polyspike-wave and spike-wave complexes. The Mayo Clinic authors also consider pattern-sensitive epilepsy to represent a distinct subtype of visually-induced reflex epilepsy, warranting routine testing in EEG recordings, and especially in patients with absence, myoclonic, or generalized tonicclonic seizures. Whereas almost $50 \%$ of the patients with pattern-sensitive epilepsy in the Mayo Clinic study achieve complete seizure remission, a group of patients with typical absence seizures triggered by photosensitivity fail to remit, in a recent report from Istanbul, Turkey (Baykan B et al. Epilepsia Jan 2005;46:159-163).

\title{
CHROMOSOMAL ABNORMALTIES WITH EPILEPSY
}

The correlation between specific chromosome abnormalties and various epilepsies was investigated by a study of 76 patients' records obtained by questionnaires distributed to members of Kyoto Multi-institutional Study Group of Pediatric Neurology. Chromosome abnormalities included the following: Down syndrome in 19 patients, Angelman syndrome (8), Prader-Willi syndrome (4), 4p-syndrome (3), and 35 other chromosome syndromes with 1-2 patients each. The severity of mental retardation correlated with the severity of epilepsy. With the exception of Angelman syndrome which showed a relatively good epilepsy prognosis, patients with severe mental retardation had a poor seizure prognosis $(\mathrm{P}<0.005)$. The type of seizure disorder showed some correlation with the developmental syndrome: febrile seizures were more frequent in patients with Angelman and Prader-Willi syndromes than with other syndromes $(\mathrm{P}<0.005)$, and were often seen with $4 \mathrm{p}$-syndrome $(2 / 3)$; status epilepticus was also characteristic of 4p-syndrome; West syndrome and focal epilepsy were common in Down syndrome; and partial seizures and a good prognosis of epilepsy were present in Klinefelter syndrome $(n=2)$. The EEG paroxysmal abnormalities in occipital regions and diffuse high voltage slow waves found in Angelman syndrome were characteristic and helpful in diagnosis. (Kumada T, Ito M, Miyajima T et al. Multiinstitutional study on the correlation between chromosomal abnormalities and epilepsy. Brain Dev March 2005;27:127-134). (Respond: Dr Tomohiro Kumada, Department of Pediatrics, Kyoto University, 54 Shogoinkawaracho, Sakyoku kyoto, Kyoto 606-8507, Japan).

COMMENT. Febrile seizures are a common feature of chromosome syndromes. In this study, the incidence of febrile seizures was higher in Angelman syndrome $(75 \%, 6 / 8)$ and Prader-Willi syndrome $(100 \%, 4 / 4)$ than with other chromosome abnormalties $(28 \%$, $18 / 64)$ or in the general population (3-4\%). The authors propose that the deleted region of chromosome 15q11-13 that is present in both Angelman and Prader-Willi syndromes may be a new locus for febrile seizures and epileptogenesis. The above article is one of a series on chromosomal aberrations and childhood epilepsies published in the same journal and 
presented at a 2003 annual meeting of the Infantile Seizure Society in Tokyo, organized by Dr Yukio Fukuyama and colleagues (see Preface. Brain Dev March 2005;27:79).

\section{NEUROLOGIC AND EEG FINDINGS IN ANGELMAN SYNDROME}

The neurological and diagnostic aspects of Angelman syndrome (AS) are reviewed by a geneticist at the University of Florida, Gainesville, FL. The prevalence of AS is $1 / 10,000$ to $1 / 20,000$. The syndrome presents in infancy with global developmental delay, microcephaly, seizures or an ataxic/hypotonic form of cerebral palsy. The facial features and general physical examination are generally normal, although a protruding tongue, strabismus, brisk deep tendon reflexes, and a happy demeanor may be present. Hypopigmentation in infants with AS due to deletion of the P pigment gene but may be overlooked. The diagnosis becomes more evident after 1 or 2 years of age, when speech does not develop, walking is impaired by severe ataxia, and seizures occur. The EEG findings can be diagnostic, with high voltage slow waves at $4-6 \mathrm{c} / \mathrm{s}$ throughout the record, $2-3 \mathrm{c} / \mathrm{s}$ slow activity in runs, especially anteriorly, and spikes or sharp waves posteriorly, provoked by eye-closure. The diagnosis is usually obvious clinically after 3 years of age and is sometimes first suggested by the parents. Behavior is often outgoing, hyperactive, hyperexcitable with excessive laughing, grabbing to engage siblings, putting objects in the mouth, and drooling. These characteristics without signs of degeneration and associated with microcephaly, seizures, and ataxia are classical. Maternally derived chromosome 15 was implicated in 1980, with microdeletion of 15q11.215q13. Subsequently, the ubiquitin ligase gene, UBE3A, located at 15q11.2, was identified as the AS gene, and 4 genetic mechanisms were involved, microdeletions being most common. The type of genetic mechanism was correlated with the severity of AS, patients with large chromosome deletions having a greater risk of seizures, microcephaly, and hypopigmentation of skin, eye and hair. The distinct behavioral syndrome and seizure patterns are related to the effects of UBE3A occurring during neuronal development. DNA methylation testing of blood is a sensitive and specific screening for 3 of the 4 genetic mechanisms. Chromosome 15 FISH analysis is necessary to distinguish which mechanism is involved. In $10-15 \%$ of cases genetic test confirmation is not possible. Genetic counseling should be offered to families of AS patients since UBE3A mutations carry a $50 \%$ recurrence risk, while common deletion cases have $<1 \%$ recurrence risk. (Williams CA. Neurological aspects of the Angelman syndrome. Brain Dev March 2005;27:88-94). (Respond: Dr Charles A Williams, Division of Genetics, Department of Pediatrics, University of Florida, PO Box 100296, Gainesville, FL 32610).

COMMENT. Clinical consensus criteria for the diagnosis of AS (Williams CA, Angelman H, Clayton-Smith J et al. Am J Med Genet 1995;56:237-238) include consistent $(100 \%)$, frequent (in more than $80 \%$ ), and associated $(20-80 \%)$ symptoms and signs. The consistent findings include: developmental delay, speech impairment, ataxia or tremor, and unique behavioral abnormalities, with frequent laughing, happy demeanor, excitability, hand flapping, hyperactivity, and short attention span. Frequent findings include: delayed head growth with microcephaly by 2 years, seizures with onset $<3$ years, and characteristic abnormal EEG. Associated findings include: flat occiput, protruding tongue, feeding and swallowing disorders, drooling, strabismus, hypopigmented skin, hair and eyes, hyperactive relexes, flexed arm posture, sleep disorder, and fascination with water. 